\title{
Compact and low-cost handheld quasibright-field linear-array probe design in photoacoustic computed tomography
}

\author{
Yuanyuan Bai \\ Bing Cong \\ Xiaojing Gong \\ Liang Song \\ Chengbo Liu
}




\title{
Compact and low-cost handheld quasibright-field linear-array probe design in photoacoustic computed tomography
}

\author{
Yuanyuan Bai, Bing Cong, Xiaojing Gong, Liang Song, and Chengbo Liu* \\ Chinese Academy of Sciences, Shenzhen Institutes of Advanced Technology, Research Laboratory for Biomedical Optics and \\ Molecular Imaging, Shenzhen, China
}

\begin{abstract}
The optimal photoacoustic probe design is the key to obtain highest imaging sensitivity in photoacoustic computed tomography. Two commonly used probe design types are dark- and bright-field designs. We proposed a design for photoacoustic probe called quasibright-field illumination and compared the performance of all three kinds of probes theoretically and experimentally. Our conclusion is that the proposed quasibright-field illumination photoacoustic probe is superior compared to the existing probe designs as demonstrated. However, each type of illumination should still have its own advantages under certain circumstances. The dark-field illumination is capable of minimizing surface interference signals and reducing their contributions to the background of deeper signals. Hence, it should perform better when imaging samples with high optical absorbance at the surface layer. The bright field may perform better under circumstance when phase distortion is less. We also designed and fabricated three kinds of probes using a single multimode optical fiber for laser energy delivery instead of fiber bundle. Single fiber probes are low cost, transmit laser energy efficiently, and are compact for easy handling. Thus, our study not only provides a method for probe design but also a guidance for cost-effective transducer array-based photoacoustic probe design and manufacturing in the future. (C) 2018 Society of Photo-Optical Instrumentation Engineers (SPIE) [DOI: 10.1117/1.JBO.23.12.121606]
\end{abstract}

Keywords: photoacoustic computed tomography; transducer array; handheld; photoacoustic probe; Monte Carlo simulation.

Paper 180412SSRR received Jun. 30, 2018; accepted for publication Sep. 4, 2018; published online Sep. 24, 2018.

\section{Introduction}

Photoacoustic (or optoacoustic) tomography (PAT) is a hybrid imaging modality that combines optical excitation with acoustic detection. The imaging technology is based on a physical phenomenon called photoacoustic effect, which describes the generation of acoustic waves by optical chromophores upon absorption of photons. In PAT, the sample of interest is usually irradiated by a short-pulsed (usually a nanosecond pulse) laser. The partial laser energy absorbed by the sample is converted into heat, which is further converted to pressure rise due to the thermal expansion. The pressure rise is propagated as acoustic waves, which is also referred to as photoacoustic waves, and is detected by an ultrasound transducer to form a PAT image of the sample. ${ }^{1}$ As a hybrid imaging method, PAT not only has the high contrast of optical imaging but also has high resolution of ultrasound detection at large imaging depth. PAT enables multiscale high-resolution imaging of biological structures, ranging in size from organelles to organs. ${ }^{2,3}$ Currently, there is a wide range of biomedical applications of PAT in the area of oncology, vascular biology, neurology, gastroenterology, dermatology, and cardiology. ${ }^{4-10}$

Photoacoustic computed tomography (PACT) is one of the mainstream photoacoustic imaging implementations of PAT imaging. It uses an ultrasound array transducer to detect photoacoustic signals from the large region of interest and reconstructs a two-dimensional or three-dimensional (3-D) photoacoustic image by processing the acquired data. Compared to other photoacoustic implementations, such as photoacoustic microscopy that is generally based on the raster scanning of a single-element ultrasound transducer, PACT has deeper imaging penetration and higher imaging speed because of the array transducer. $^{3}$ In addition, PACT imaging system can be built on a clinical ultrasound imaging platform enabling the potential of translating PACT for clinical applications. Many research groups have been investigating PACT system implementation, ${ }^{11-14}$ and the main differentiator among these systems is the design of the photoacoustic probes, i.e., the integration scheme employed to combine light and ultrasound array transducer. There are several photoacoustic probe designs in the literature ${ }^{11,15-16}$ and the most common is called dark-field illumination design and is shown in Fig. 1(a). In this design, the light path is positioned obliquely on one or both sides of the ultrasound array transducer to shed laser energy onto the sample surface. However, since the light enters the imaging sample from the side of the ultrasound transducer, the light delivery efficiency of this system into the biological scattering medium (i.e., light delivered to the ultrasound transducer's field of view) is low, resulting in low photoacoustic signal. In addition, due to the oblique incidence of the light, the imaging region is only confined to the deeper region and the shallower region is not imaged. Thus, to achieve high signal-to-noise ratio (SNR) throughout the imaging plane, the incident light should be shed on the sample surface directly underneath the ultrasound transducer. Previously, we proposed a bright-field illumination photoacoustic probe design to image both shallower and deeper regions of interest [as shown in Fig. 1(b)]. ${ }^{17}$ In this design, the laser energy delivered by a single fiber on the side of the 
(a)

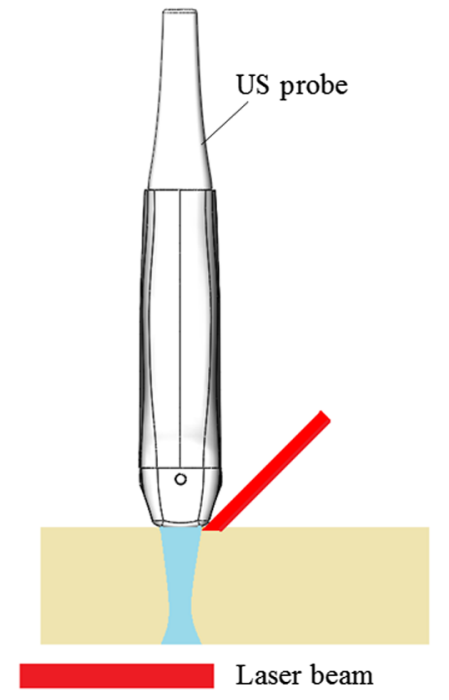

(b)

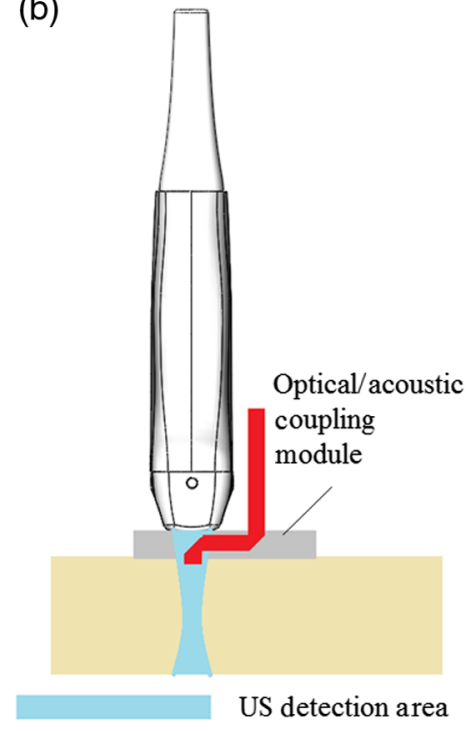

(c)

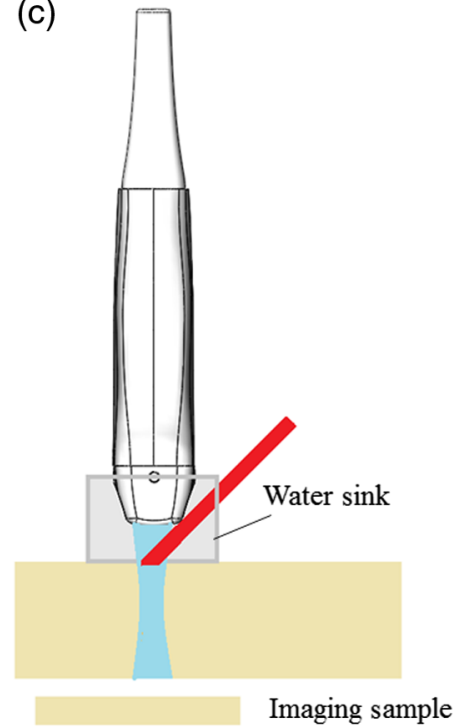

Fig. 1 Schematic of light illumination of variant photoacoustic probe designs: (a) dark-, (b) bright-, and (c) quasibright-field illuminations. US, ultrasound.

ultrasound transducer is shaped into a rectangular beam with a set of optical lenses and guided to the sample surface underneath the ultrasound transducer using a custom-made optical/acoustic coupling module. Compared to dark-field illumination design, this design enables perfect overlapping of optical excitation and acoustic detection at the sample surface (also referred as coaxial optical and acoustic design) resulting in a highly efficient light distribution in the ultrasound detection region. Compared to the dark-field design, the bright-filed design achieves higher SNR and increased imaging depth. One drawback of this design, however, is the attenuation of ultrasound signals in the optical/acoustic coupling module made of polymethyl methacrylate materials. More importantly, the acoustic impedance mismatch between the optical/acoustic coupling module and the surrounding medium (water or ultrasound gel) results in severe phase distortion of the photoacoustic signal wavefront, leading to poor image reconstruction. Although several advanced reconstruction algorithms ${ }^{18-20}$ have been proposed to restore the phase distortion, they have been successful partially, thus limiting the imaging sensitivity of this photoacoustic probe.

To overcome the above-mentioned limitations of the existing probe types, we are proposing a third type of probe design in this article called quasibright-filed illumination design as shown in Fig. 1(c). This probe design combines the advantages of darkand bright-field illumination designs mentioned above while addressing the limitations. In this approach, the excitation light is incident obliquely to the sample surface, sitting directly underneath the ultrasound transducer, to enable highly efficient light distribution in the ultrasound detection region. The ultrasound transducer is coupled to the sample surface with materials whose acoustic impedance is similar to biological tissues, such as water or ultrasound gel, to reduce the transmission loss and eliminate the wavefront distortion of photoacoustic signals.

In this study, we compare the performance of the dark- and bright-field probe designs with our proposed quasibright-field illumination probe design using theoretical simulation. ${ }^{21}$ In addition, unlike the previous publication where experimental validations were missing, ${ }^{17}$ in this study, we also validate all three probes performance experimentally using both phantom and ex-vivo biological samples. The details of our simulation as well as experimental evaluation are presented in the subsequent sections. The performance of all three photoacoustic probe types is assessed for biomedical research as well as clinical application potentials.

\section{Materials and Methods}

\subsection{Photoacoustic Probe Design and Fabrication}

Detailed configurations of all three photoacoustic probe designs and the real photos of the fabricated probes are shown in Fig. 2. The main components in all three designs include an optical fiber with a $1500-\mu \mathrm{m}$ core diameter, two cylindrical lenses (plano-convex and plano-concave cylindrical lens, AYL1210B and LK1006L1-B, Thorlabs, Newton, New Jersey), a 128element ultrasound linear-array transducer $(7 \mathrm{MHz}$, Blatek, Pennsylvania), and a 3-D-printed shell holder. The two cylindrical lenses shown in Figs. 2(a), 2(b), and 2(c) are utilized to reshape the excitation light coming out of the optical fiber into a narrow $38 \times 2 \mathrm{~mm}$ rectangular pattern $(38 \mathrm{~mm}$ is the length of the ultrasound transducer). The 3-D-printed shell holder provides mechanical support and protection to all optical and acoustic components. The designs of quasibright- and darkfield illumination probe are similar except with respect to illumination technique. In the dark-field illumination design [Fig. 2(a)], the light illuminates the sample surface obliquely from the side of the ultrasound probe. For the quasibrightfield illumination design [Fig. 2(c)], the excitation light is guided to the sample surface right beneath the ultrasound transducer. A hollow plastic tube is fitted at the bottom of the ultrasound transducer to enable quasibright-field illumination. The tube is transparent and filled with water to allow acoustic transmission to the surface underneath the ultrasound transducer. The bottom of the tube is sealed with a thin polystyrene membrane to minimize penetration loss of photoacoustic signals. The bright-field illumination design is shown in Fig. 2(b). In this design, a custom-made optical/acoustic coupling module guides 
(a) US probe

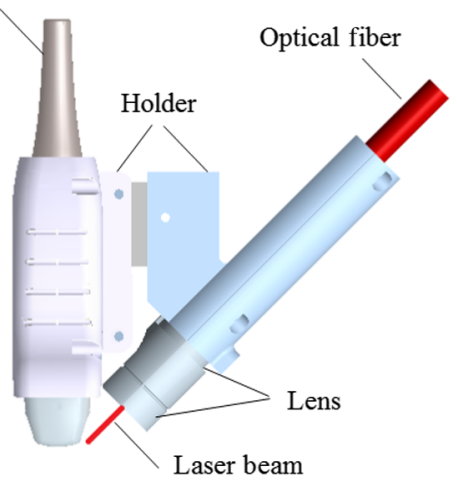

(d)

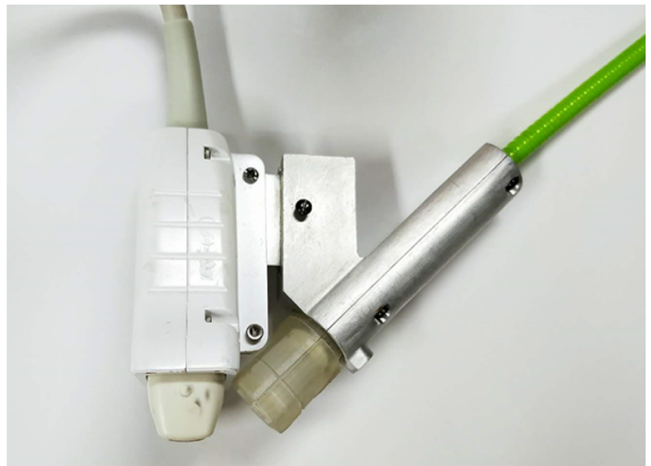

(b)

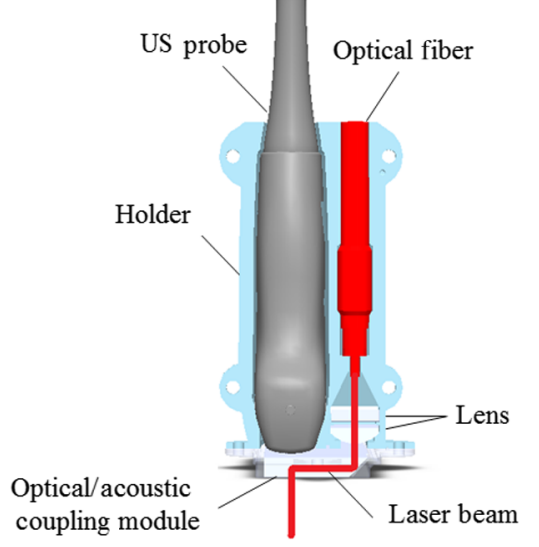

(e) (c) US probe

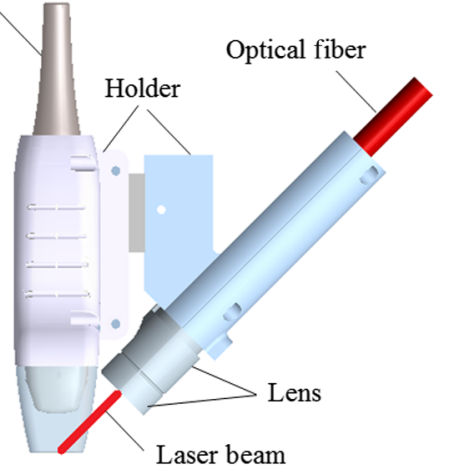

(f)

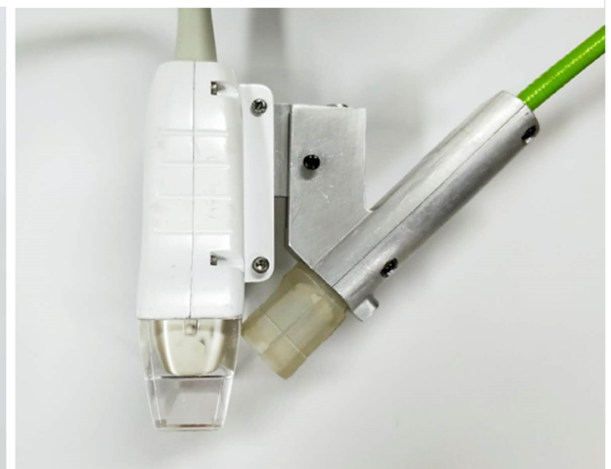

Fig. 2 (a)-(c) Schematic design and (d)-(f) real photo of the three photoacoustic probes. (a) and (d) Dark-, (b) and (e) bright-, and (c) and (f) quasibright-field illumination probes. US, ultrasound.

the excitation light to the region underneath the ultrasound transducer [as indicated by the red line in Fig. 2(b)]. The excited photoacoustic signals from the imaging sample transmit through the optical/acoustic coupler to be detected by the ultrasound transducer. Coaxial optical excitation and acoustic detection are achieved in the bright-field illumination probe design. The detailed information on this probe can be found in our previous publication. ${ }^{17}$

\subsection{Monte Carlo Simulation of Photon Propagation}

To compare the performance of all three photoacoustic probe designs, discussed in Sec. 2.1, we simulated photon propagating into the semi-infinite scattering medium, which mimics the biological tissues, using Tracepro ${ }^{\mathrm{TM}}$ software (Lambda Research Corporation, Littleton, Massachusetts). The light illumination patterns for all three designs at the sample surface are shown in Fig. 3. The size of the laser beam was set to $38 \times 2 \mathrm{~mm}$. For the dark- and quasibright-field illumination designs, the incident angle of the laser beam was set at $45 \mathrm{deg}$ (the laser beam on the sample surface is $\sim 2.83 \mathrm{~mm}$ in width). For the bright-field illumination design, the incident angle was set at 0 deg (i.e., the laser beam enters the sample vertically). The centers of the laser beam and the ultrasound transducer detection area are overlapped for the bright-field and quasibright-field on the sample surface, respectively, and for the dark-field, (a)

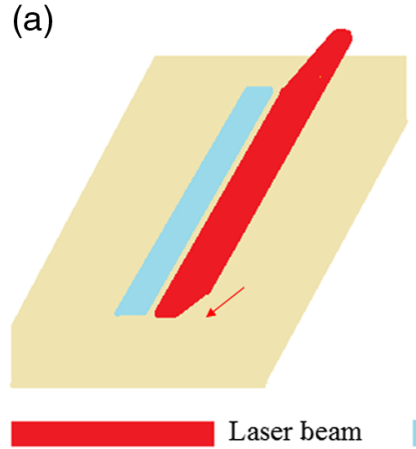

(b)

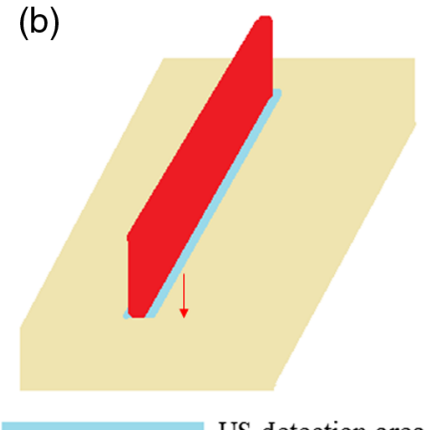

(c)

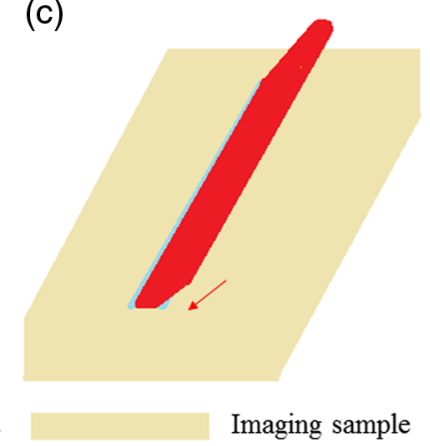

Fig. 3 Light illumination patterns at the imaging sample surface for the (a) dark-, (b) bright-, and (c) quasibright-field photoacoustic probes. US, ultrasound 
the centers were $7.5 \mathrm{~mm}$ apart. The above parameters were derived from the actual size of the ultrasound transducer and the true optical excitation condition of the photoacoustic probes. The optical absorption coefficient, optical scattering coefficient, anisotropic factor, and refractive index of the imaging sample were set to $0.1 \mathrm{~cm}^{-1}, 200 \mathrm{~cm}^{-1}, 0.9$, and 1.37 , respectively, which approximate the real conditions of the biological tissues. ${ }^{22}$ The total flux of the incident light was set to the same value in all simulations. Furthermore, to investigate the influence of the optical incidence angle on the laser fluence distribution, we performed the simulation at other angles $(5 \mathrm{deg}$, $20 \mathrm{deg}, 35 \mathrm{deg}$, and $50 \mathrm{deg}$ ) for both the dark- and quasibright-field illuminations.

\subsection{Photoacoustic System}

To evaluate the performance of the three photoacoustic probes, we built a photoacoustic imaging system using Verasonics Vantage ultrasound platform (Verasonics, Inc., Kirkland, Washington). The schematic of the hybrid system is shown in Fig. 4. An optical parametric oscillator laser (Innolas $\mathrm{GmbH}$, Bonn, Germany) emitting short (<10 ns) laser pulses was used to provide optical excitation in a tunable wavelength range between 690 and $900 \mathrm{~nm}$. The laser pulse was attenuated by a neutral density filter and then focused by a convex lens for coupling into a multimode optical fiber with $1500-\mu \mathrm{m}$ core diameter. At the distal end of the fiber, the excitation laser was integrated with a 128-element linear-array ultrasound transducer (7 MHz, Blatek, Pennsylvania). The detected photoacoustic signals from the ultrasound transducer were transferred to the 128-channel data acquisition board integrated inside the Verasonics imaging platform for further processing. The Q-switch output from the laser source synchronized the laser firing and data acquisition, thus enabling the collection of photoacoustic data at a frame rate equal to the pulse repetition frequency of the laser $(20 \mathrm{~Hz})$. The standard back-projection algorithm was applied to reconstruct the tomographic photoacoustic image. ${ }^{17}$ Following the acquisition of the photoacoustic signals, our hybrid platform was used to acquire ultrasound image of the area underneath the transducer to coregister photoacoustic and ultrasound images.

\subsection{Phantom Experiment}

The focus of our study is to compare the sensitivity of the photoacoustic probes at various depths due to the differences in optical illumination designs. Hence, the depth-dependent sensitivity of the ultrasound transducer should be compensated in the received photoacoustic signals, before comparison. To determine this depth-dependent compensation, we performed phantom experiment of the ultrasound transducer at different depths. A black suture was placed horizontally in a $5 \times 5 \times 4 \mathrm{~cm}$ plastic water sink under the ultrasound transducer and a laser beam was used to irradiate the suture from the horizontal direction. The suture remained fixed in the water while the ultrasound transducer was moved to obtain the photoacoustic signals of the suture at different imaging depths. The SNR of photoacoustic signals at various depths (i.e., the acoustic detection sensitivity of the ultrasound transducer) was quantified and used to compensate for the variance in ultrasound detection sensitivity for all the subsequent experiments.

The performance of three photoacoustic probes was evaluated by imaging a series of black sutures embedded in agar phantom with $1 \%$ fat emulsion at different depths. An 800nm wavelength was used for photoacoustic signal excitation and the light energy was $\sim 15 \mathrm{~mJ}$, which corresponds to a laser fluence of $9.8 \mathrm{~mJ} / \mathrm{cm}^{2}$ at the surface of the imaging sample. Both photoacoustic and ultrasound B-scan images were acquired for all three probes.

\subsection{Ex-vivo Canine Prostate Imaging Experiment}

To validate the performance of photoacoustic probes for potential preclinical and clinical applications, an excised prostate of

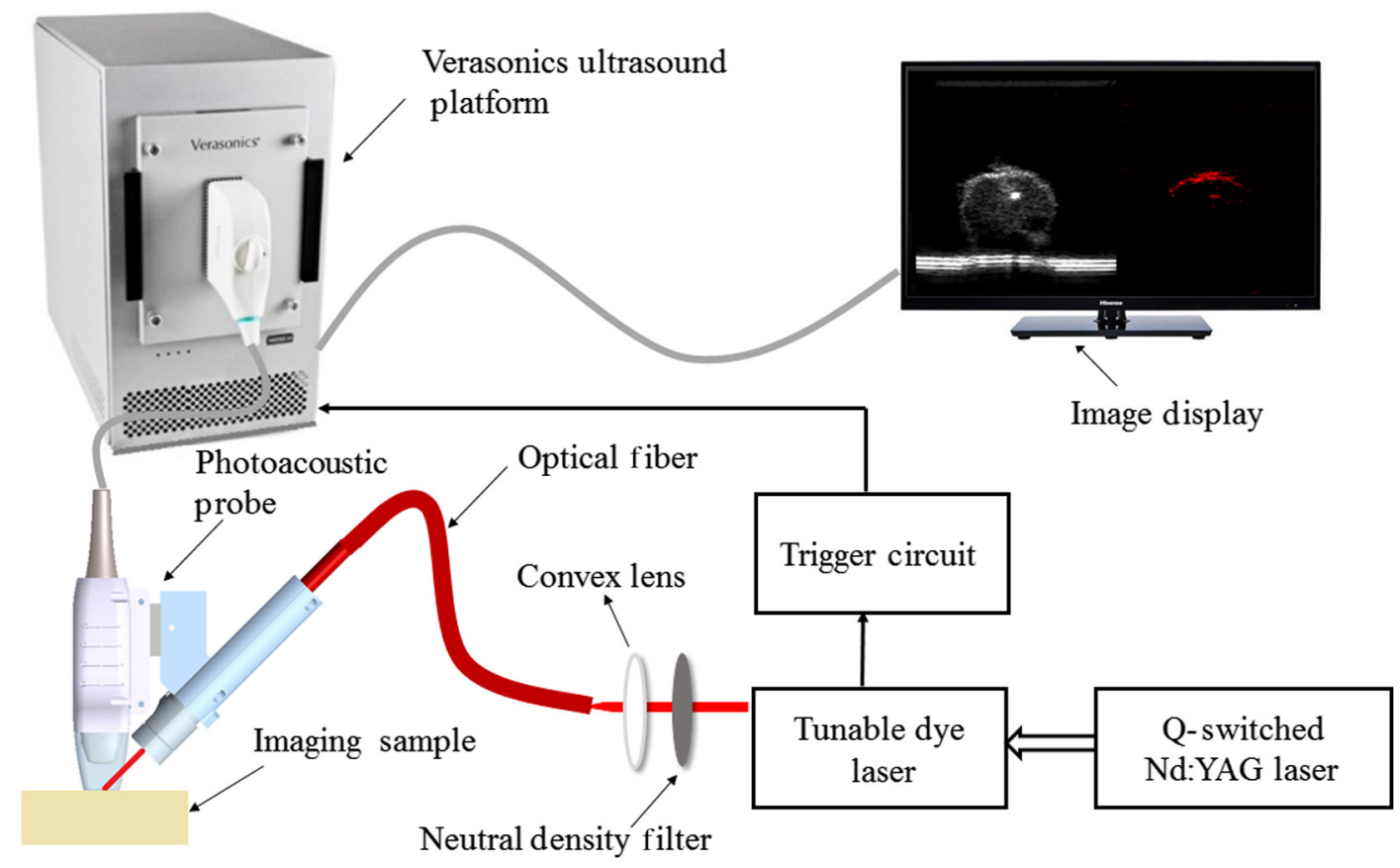

Fig. 4 Schematic of the photoacoustic imaging system. 
(a)

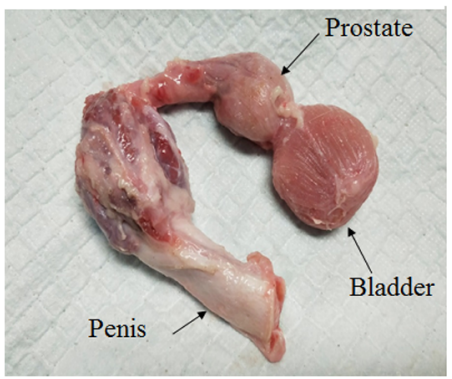

(b)

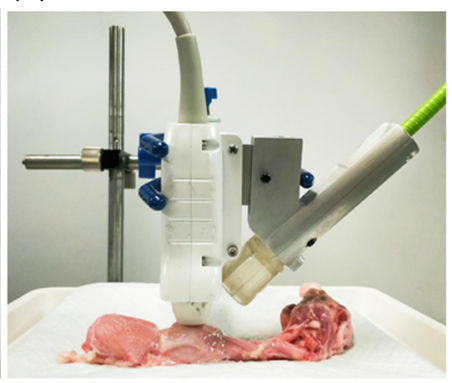

(c)

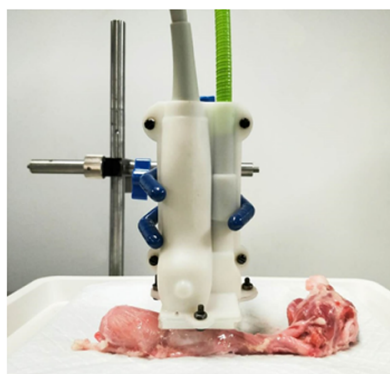

(d)

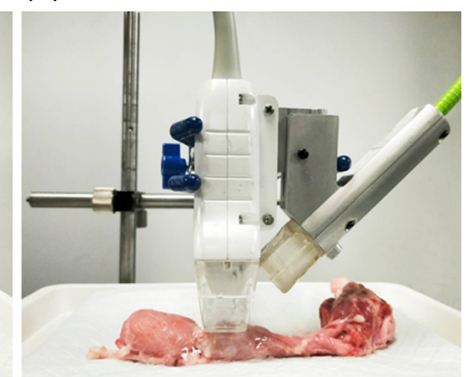

Fig. 5 (a) Photo of ex-vivo canine prostate and experimental setup of photoacoustic imaging of the excised canine prostate with the (b) dark-, (c) bright-, and (d) quasibright-field illumination probes.

beagle dog was imaged with all three probes. A blood lump was formed by injecting blood into the prostate to mimic the tumor condition in the prostate. The photoacoustic probes were placed on top of the prostate during the imaging process as shown in Fig. 5. Ultrasound gel was filled between the ultrasound transducer and the prostate to provide acoustic transmission coupling. The 800-nm wavelength, close to a localized optical absorption peak of hemoglobin, was utilized for photoacoustic signal excitation. The laser fluence on the sample surface was about $9.8 \mathrm{~mJ} / \mathrm{cm}^{2}$, well below the ANSI safety limit $\left(30 \mathrm{~mJ} / \mathrm{cm}^{2}\right){ }^{23}$ The experimental setup is shown in Fig. 5 . To evaluate the probes at larger depths, we also added chicken breast layers of two different thicknesses $(5$ and $10 \mathrm{~mm}$ ) on top of the prostate and acquired the photoacoustic and ultrasound images. All the animal samples were handled according to the protocol approved by the Animal Study Committee of Shenzhen Institutes of Advanced Technology, Chinese Academy of Sciences.

\section{Results and Discussion}

\subsection{Simulation}

The simulation result of photon propagating into the tissuemimicking scattering medium is shown in Fig. 6 for all three photoacoustic probes. Figures 6(a)-6(f) show the laser fluence of dark-field illumination at depths of $0.1,5,10,15,20$, and $25 \mathrm{~mm}$, respectively, below the sample surface. Figures $6(\mathrm{~g})$ 6(l) and 6(m)-6(r) show same results for the bright- and quasibright-field illuminations, respectively. The white-dashed rectangle in each figure indicates the ultrasound detection region for that depth. Figure 6(s) shows the quantitative comparison of the laser fluence in the ultrasound detection area for all three photoacoustic probes at all depths. The solid lines in the figure are the fitting curves of the quantification. Compared to the dark-field illumination, the laser fluence is significantly higher for the quasibright- and bright-field illumination designs when the imaging depth is $<15 \mathrm{~mm}$. When the depth is increased to $15 \mathrm{~mm}$ or more, the laser fluence of the quasibright field and bright field remains $2 \times$ times higher than that of darkfield illumination. This is illustrated in Fig. 6(t), which is the zoomed-in view of the black-dashed area in Fig. 6(s). When we compared the quasibright- and bright-field illumination designs, we found that the laser fluence of the quasibright field is slightly lower for the imaging depth between 0 and $5 \mathrm{~mm}$. This is mainly because the size of the laser beam projected on the sample surface was enlarged due to oblique incidence. When the imaging depth is increased to $5 \mathrm{~mm}$ and above, the fluence of the two designs tends to get equalized.

The simulation results for different incidence angles ( $5 \mathrm{deg}$, $20 \mathrm{deg}, 35 \mathrm{deg}$, $45 \mathrm{deg}$, and $50 \mathrm{deg}$ ) for dark- and quasibrightfield illuminations are shown in Figs. 6(u) and 6(v), respectively. For dark-field illumination, laser fluence is higher for almost all simulated imaging depths when incidence angle is large (i.e., when the light is flatter) although only depths within upper $25 \mathrm{~mm}$ are simulated. On the other hand, for the quasibrightfield illumination, the laser fluence is higher at all imaging depths when the incidence angle is small (i.e., the light is more vertical). Although more laser energy is delivered to the ultrasound detection region at smaller incidence angle in quasibright-field illumination, the shallower region of the ultrasound detection will be sacrificed more to enable the laser beam to be shed on the sample surface directly underneath the ultrasound transducer. Since $45 \mathrm{deg}$ is a good balance between the laser energy delivered and the ultrasound detection, we used this setting for our probe.

\subsection{Phantom Experiment}

The calculated photoacoustic SNR of the black suture target at different imaging depths is shown in Fig. 7. A fitting curve in solid line is drawn through the data as shown in Fig. 7(b). Since the laser irradiation is the same for all the depths of measurements, the variation in the SNR is mainly due to the varying sensitivity of the ultrasound transducer at different depths. For our subsequent experiments, we used this curve to compensate the acoustic detection at different depths.

The B-scan photoacoustic images of multiple black sutures in agar phantom containing $1 \%$ fat emulsion are shown in Figs. 8(a)-8(d) using all three photoacoustic probes. For the bright-field probe, two photoacoustic images were reconstructed using conventional back-projection algorithm [Fig. 8(b)] and fast marching method (FMM)-based back-projection algorithm [Fig. 8(c)] as reported in our previous publication. ${ }^{17}$ The FMM method calculates the path and arrival time of the acoustic signal to each element of the ultrasound transducer and includes the delay directly into the back-projection algorithm to reconstruct each pixel in the image. The SNR quantification results for all the images are shown in Fig. 8(e) with the solid curve line fitting the quantification values. Compared to the bright- and quasibright-field illumination probes, the dark-field probe has much lower imaging sensitivity at the shallower imaging depths resulting in almost no photoacoustic signals for the top black sutures [white circles in Fig. 8(a)]. This is mainly due to the low laser fluence at the shallower region of the imaging sample 

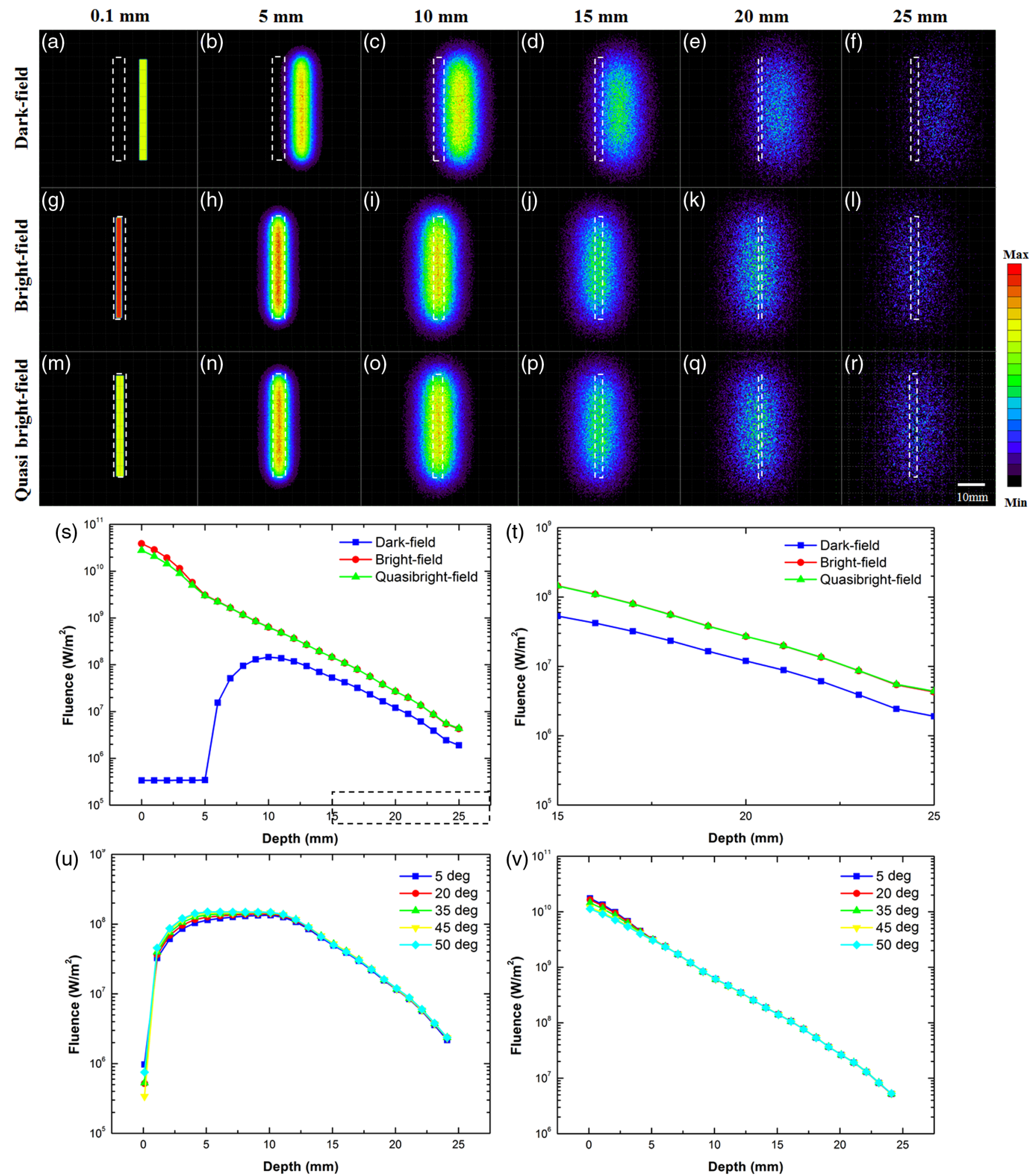

Fig. 6 Simulation results of laser fluence at different depths in scattering medium for the (a)-(f) dark-, (g)-(l) bright-, and (m)-(r) quasibright-field illumination photoacoustic probe designs. (s) Quantitative comparison of laser fluence in the ultrasound detection area at different depths for the three photoacoustic probe designs. Blue line: dark-field illumination, red line: bright-field illumination, and green line: quasibright-field illumination. (t) Zoomed-in view of the black rectangle in (s). Quantitative analysis of the simulation results for different incidence angles (5 deg, $20 \mathrm{deg}, 35 \mathrm{deg}, 45 \mathrm{deg}$, and $50 \mathrm{deg}$ ) in (u) dark- and (v) quasibright-field illuminations. 

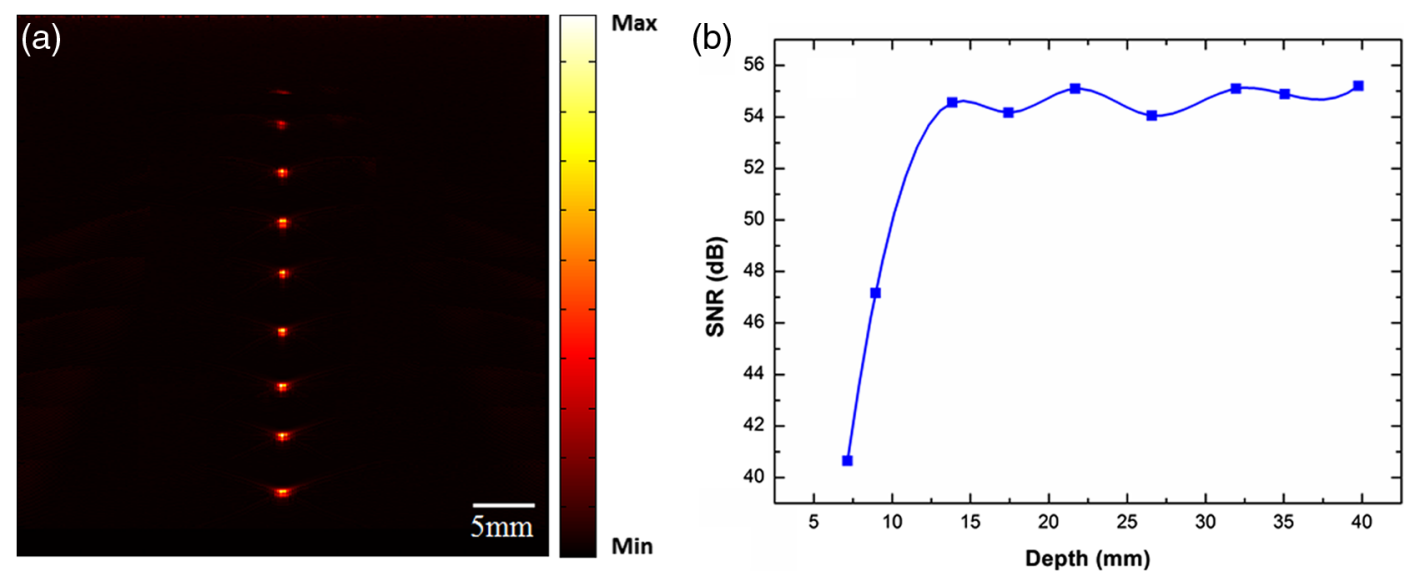

Fig. 7 (a) B-scan photoacoustic image of black suture at different depths in water and (b) the calculated SNR corresponding to each depth. The solid line is the fitting curve of the calculation.

(a)

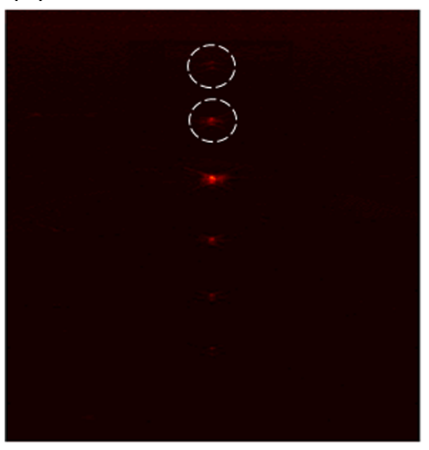

(c)

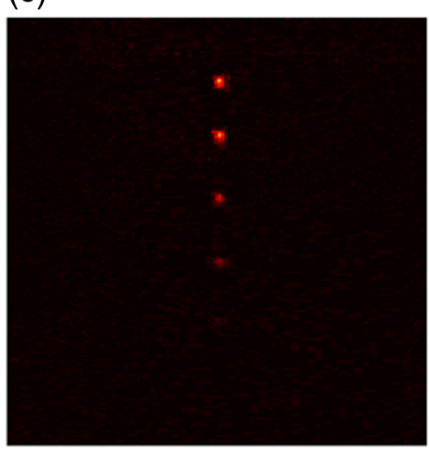

(b)

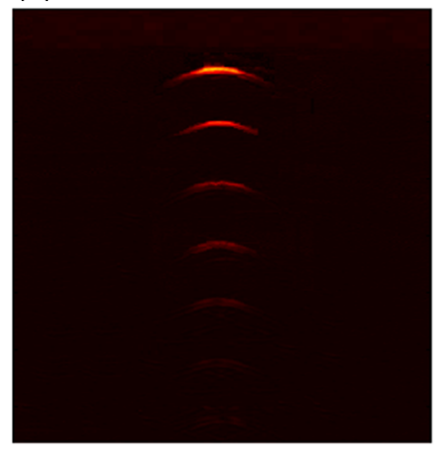

(d)

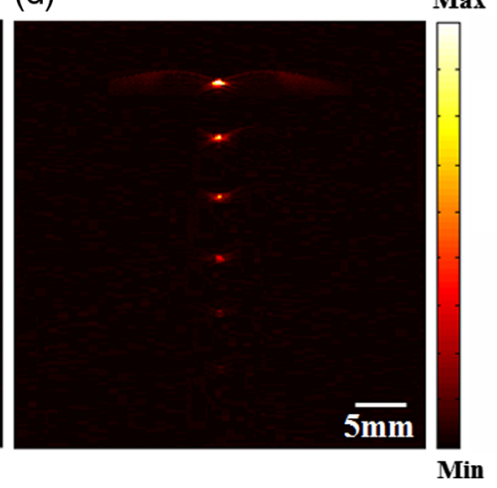

(e)

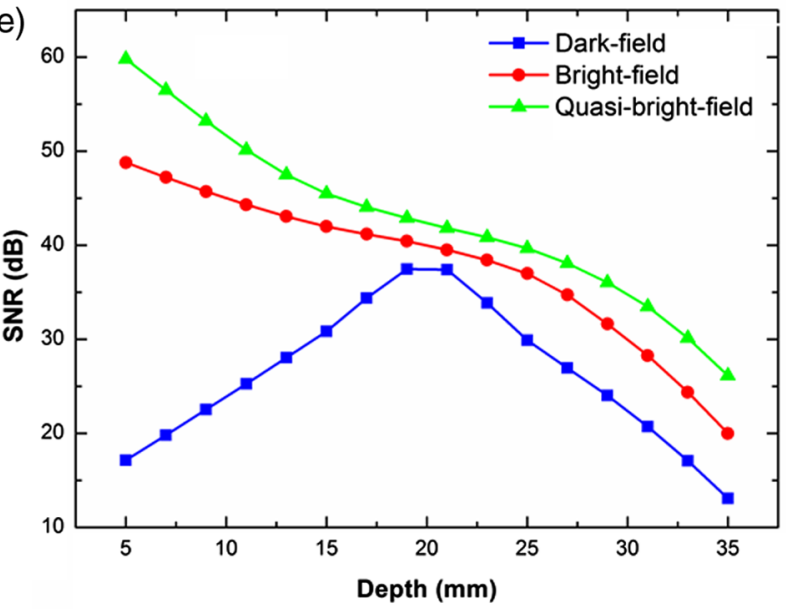

Fig. 8 (a)-(d) B-scan photoacoustic images of multiple black sutures in agar phantom containing 1\% fat emulsion when using different photoacoustic probes and image reconstruction methods. (e) The SNR quantification of all the images. The solid lines are the fitting curves of the calculation.

for this probe design, similar to the simulation results in Fig. 6. The photoacoustic imaging results agree well with the simulation results for all imaging depths. The reconstructed B-scan image without and with phase correction is shown in Figs. 8(b) and 8(c) for the bright-field illumination probe, respectively. The phase correction of the acoustic wavefront was accomplished using FMM reconstruction method as reported in our previous publication. Although the SNR and resolution of the image are improved significantly after phase correction, the quality of the image with the bright-field illumination probe is still lower than quasibright-field illumination probe, indicating the phase distortion induced by the optical/acoustic coupling module is difficult to compensate completely. Among all the three probe designs, the quasibright-field probe has the highest SNR at all imaging depths as shown in Fig. 8(e). Although the laser fluence at the shallower depth in the quasibright-field illumination design is lower than that of the bright-field design, as shown in Fig. 6, the sensitivity of the photoacoustic probe is better for the quasibright-field illumination. This is because significant ultrasound attenuation and wavefront distortion exist in the optical/acoustic coupling module of the bright-field illumination probe. Our results indicate that the quasibright-field illumination 

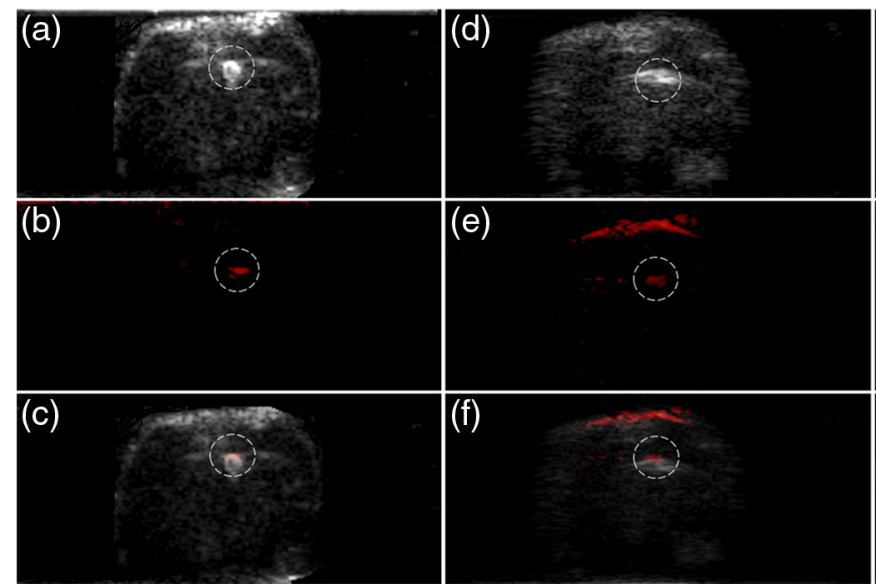
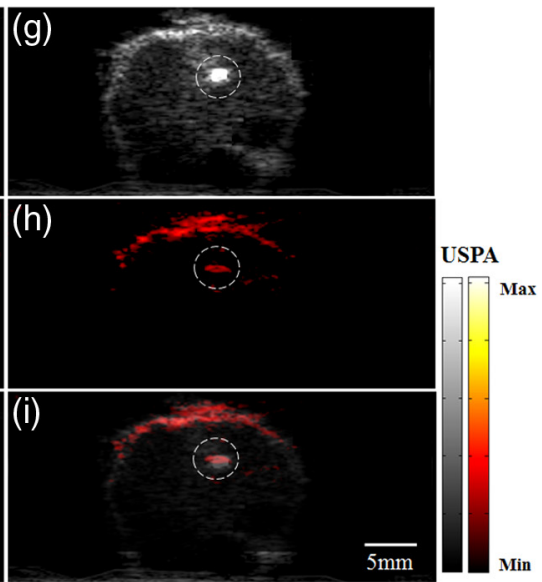

Fig. 9 B-scan ultrasound, photoacoustic, and their merged images of the beagle dog prostate when imaged with the (a)-(c) dark-, (d)-(f) bright-, and (g)-(i) quasibright-field illumination photoacoustic probes.

probe design is better suited for array transducer-based photoacoustic probe fabrication compared to other designs.

\subsection{Ex-vivo Prostate Imaging Experiment}

To assess the potential of photoacoustic probes for preclinical and clinical application, an ex-vivo prostate of a beagle dog was imaged with all three probes after injecting the blood to mimic the tumor condition. The upper surface of the prostate was also stained by the blood during the injection of blood into the prostate. The B-scan photoacoustic, ultrasound, and their merged images of the prostate are shown in Fig. 9 for all three probe designs. The surface of the prostate is at 2-, 20-, and 22-mm depth for the dark-, bright-, and quasibrightfield probes, respectively. The tumor-mimicking blood lump in the prostate is circled with white-dashed lines in the images. Similar to the phantom results, the quasibright-field illumination probe has the highest photoacoustic signal among all three probes. For the bright- and quasibright-field illumination probes, both the upper and the deeper tumor-mimicking region of the prostate can be clearly visualized in the photoacoustic images. However, when using the dark-field illumination probe, only the deeper tumor-mimicking region can be visualized with relatively low sensitivity indicating no laser fluence at
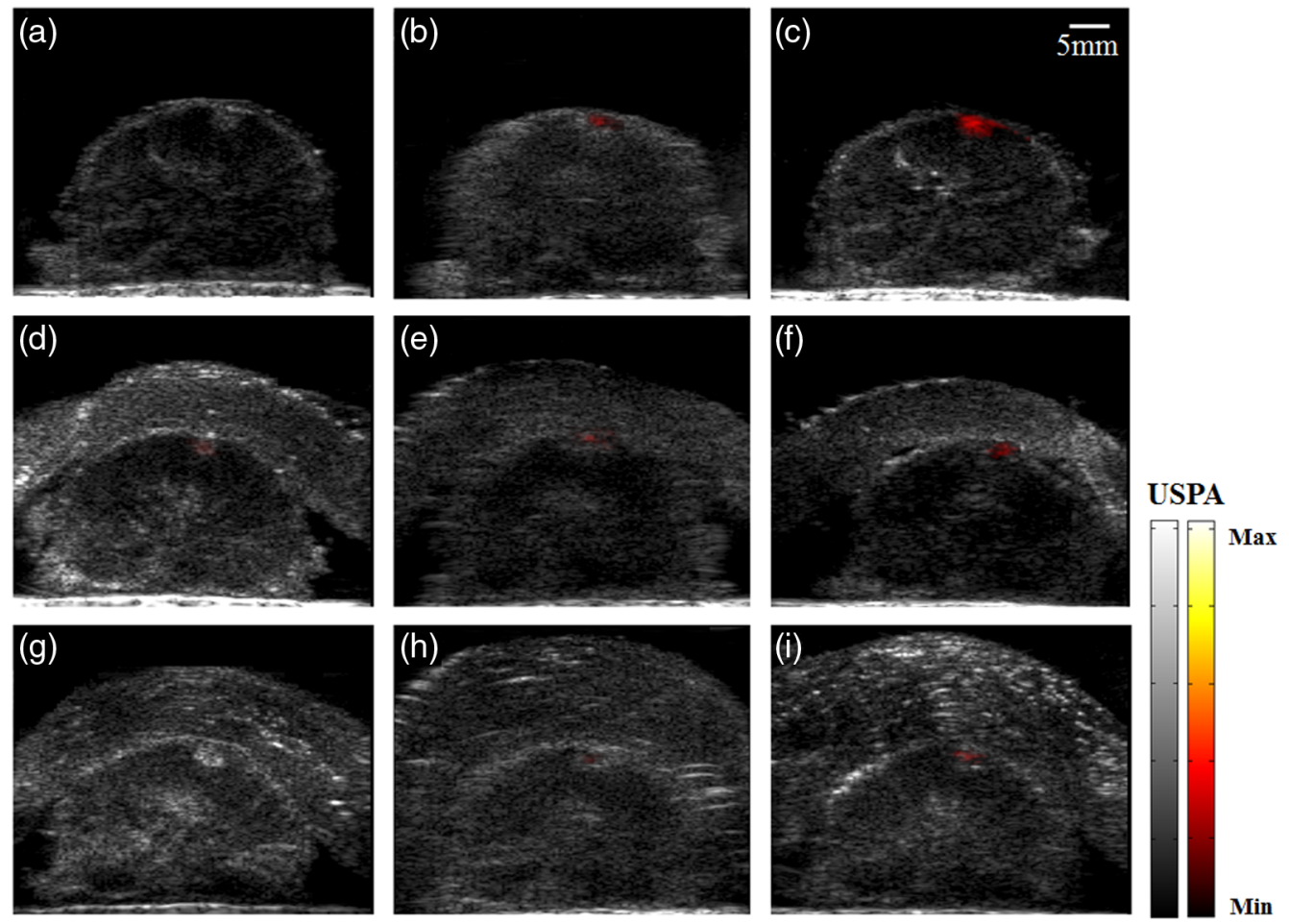

Fig. 10 B-scan ultrasound and photoacoustic merged images of the beagle dog prostate acquired with the (a), (d), (g) dark-, (b), (e), (h) bright-, and (c), (f), (i) quasibright-field illumination photoacoustic probes when covered with no chicken breast layer (a)-(c), 5-mm chicken breast layer (d)-(f), and 10-mm chicken breast layer (g)-(i). 
the shallower region and lower laser fluence in the deeper region. These imaging results also indicate that when using dark-field illumination probe design for in-vivo application, important biological features could be missed. The coregistered tumor site in the merged photoacoustic and ultrasound images confirms that, while there are advantages and disadvantages in each method, all three probes could still be used for detecting blood vascularized tumors in real clinical condition when they are at appropriate depths.

Figure 10 shows the merged photoacoustic and ultrasound images for each probe when the prostate is covered with no chicken breast layer $(\mathrm{a}-\mathrm{c}), 5-\mathrm{mm}$ chicken breast layer $(\mathrm{d}-\mathrm{f})$, and $10-\mathrm{mm}$ chicken breast layer $(\mathrm{g}-\mathrm{i})$. For this experiment, blood was injected into the upper layer of the prostate. The SNR for the three probes when covered with different chicken breast layers $(0,5$, and $10 \mathrm{~mm})$ are: $0,19.8$, and $0 \mathrm{~dB}$ for the dark-field probe; $60.49,40.29$, and $18.46 \mathrm{~dB}$ for the bright-field probe; and $66.8,46.49$, and $28.63 \mathrm{~dB}$ for the quasibright-field probe, respectively. The quasibright-field probe has the highest SNR at all the imaging depths when compared with other two probes.

All three photoacoustic probes were proposed and manufactured in this study based on a linear-array ultrasound transducer. Compared with the previously reported similar probes, such as the dark-field illumination probes that mostly used fiber bundles to deliver laser energy, a single multimode optical fiber was adopted in this study for laser energy delivery in all three probes. The laser beam out of the fiber is reshaped into the rectangular size with two cylindrical lenses to accommodate with the acoustic detection of the ultrasound transducer. The single fiber-based laser delivery scheme brings the following benefits: (1) the photoacoustic probes are more compact and light-weighted compared to those using fiber bundles, making the probes more user friendly for handling. (2) Higher optical transmission efficiency can be guaranteed with single fiber compared to fiber bundle. (3) The cost of the single fiber is generally much less than that of the fiber bundle. Thus, our proposed design would reduce the cost of the system while making it compact and easy to use.

\section{Conclusion}

Three handheld linear-array photoacoustic probes with different optical illumination designs were proposed and manufactured in this study. The performance of the probes was compared with theoretical simulation and validated with phantom and ex-vivo prostate imaging. It is to be noted that although the proposed quasibright-field illumination photoacoustic probe is superior compared to the existing probe designs as demonstrated in this study, each type of illumination should have its own advantages under certain circumstances. The dark-field illumination is capable of minimizing surface interference signals and reducing their contributions to the background of deeper signals. Hence, dark field should perform better when imaging samples with high optical absorbance at the surface layer. In some other cases, bright field may also perform better, e.g., if phase distortion is less. Thus, based on the imaging need, any of these three probe designs could be used, whereas in majority of the cases we expect our proposed probe design would be most optimal. Tilting of the dark-field probe is also a good option to be considered in many cases, which will allow more laser energy to be delivered to the ultrasound transducer detection area, although the titling angle of the probe may not be allowed to be very large to guarantee good acoustic coupling between the probe and the sample. The designs of the three photoacoustic probes in this study are all based on single fiber, which would make all these probes low-cost and compact thus improving the system implementation. In the future, we expect the user would have access to all these three probe types and would use the one appropriate for the specific application. To conclude, in the process of proposing a probe design, we also presented guidance for evaluating the transducer array-based photoacoustic probe design and fabrication for the future systems.

\section{Disclosures}

The authors declare that there are no conflicts of interest related to this article.

\section{Acknowledgments}

The authors gratefully acknowledge the following funds: the National Natural Science Foundation of China (NSFC) Grant Nos. 91739117, 81522024, 81427804, 81430038, and 61475182; the National Key Basic Research (973) Program of China Grant Nos. 2014CB744503 and 2015CB755500; the Guangdong Natural Science Foundation Grant No. 2014A030312006; the Shenzhen Science and Technology Innovation Grant Nos. JCYJ20170413153129570, JCYJ20160531175040976, and JCYJ20160608214524052; the Guangzhou Science and Technology Innovation Grant No. 201604020144; and the Chinese Academy of Sciences Grant No. GJJSTD20180002.

\section{References}

1. L. V. Wang and H. Wu, Biomedical Optics: Principles and Imaging, John Wiley \& Sons, New Jersey (2012).

2. L. V. Wang, "Multiscale photoacoustic microscopy and computed tomography," Nat. Photo. 3(9), 503-509 (2009).

3. L. V. Wang and S. Hu, "Photoacoustic tomography: in vivo imaging from organelles to organs," Science 335(6075), 1458-1462 (2012).

4. K. S. Valluru, K. E. Wilson, and J. K. Willmann, "Photoacoustic imaging in oncology: translational preclinical and early clinical experience," Radiology 280(2), 332-349 (2016).

5. A. Taruttis et al., "Optoacoustic imaging of human vasculature: feasibility by using a handheld probe," Radiology 281(1), 256-263 (2016).

6. B. Soetikno et al., "Vessel segmentation analysis of ischemic stroke images acquired with photoacoustic microscopy," Proc. SPIE 8223, 822345 (2012).

7. J. M. Yang et al., "Simultaneous functional photoacoustic and ultrasonic endoscopy of internal organs in vivo," Nat. Med. 18(8), 1297-1302 (2012).

8. B. Zabihian et al., "In vivo dual-modality photoacoustic and optical coherence tomography imaging of human dermatological pathologies," Biomed. Opt. Express 6(9), 3163-3178 (2015).

9. K. Jansen et al., "Intravascular photoacoustic imaging of human coronary atherosclerosis," Opt. Lett. 36(5), 597-599 (2011).

10. K. Sivasubramanian et al., "Hand-held, clinical dual mode ultrasoundphotoacoustic imaging of rat urinary bladder and its applications," J. Biophotonics 11(5), e201700317 (2018).

11. C. Kim et al., "Handheld array-based photoacoustic probe for guiding needle lymph nodes," J. Biomed. Opt. 15(4), 046010 (2010).

12. L. G. Montilla et al., "Real-time photoacoustic and ultrasound imaging: a simple solution for clinical ultrasound systems with linear arrays," Phys. Med. Biol. 58(1), N1-N12 (2013).

13. K. Daoudi et al., "Handheld probe integrating laser diode and ultrasound transducer array for ultrasound/photoacoustic dual modality imaging," Opt. Express 22(21), 26365-26374 (2014).

14. A. Needles et al., "Development and initial application of a fully integrated photoacoustic micro-ultrasound system," IEEE Trans. Ultrason. Ferroelectr. Freq. Control 60(5), 888-897 (2013). 
15. T. N. Erpelding et al., "Sentinel lymph nodes in the rat: noninvasive photoacoustic and US imaging with a clinical US system," Radiology 256(1), 102-110 (2010).

16. K. Sivasubramanian et al., "Optimizing light delivery through fiber bundle in photoacoustic imaging with clinical ultrasound system: Monte Carlo simulation and experimental validation," J. Biomed. Opt. 22(4), 041008 (2016).

17. $\mathrm{M}$. Li et al., "Linear array-based real-time photoacoustic imaging system with a compact coaxial excitation handheld probe for noninvasive sentinel lymph node mapping," Biomed. Opt. Express 9(4), 1408-1422 (2018).

18. M. S. Hassouna and A. A. Farag, "Multi-stencils fast marching methods: a highly accurate solution to the Eikonal equation on Cartesian domains," IEEE Trans. Pattern Anal. Mach. Intell. 29(9), 1563-1574 (2007).

19. T. Wang and Y. Jing, "A fast marching method based back projection algorithm for photoacoustic tomography in heterogeneous media," Phys. preprint arXiv:1501.03869 (2015).

20. T. Wang and Y. Jing, "Transcranial ultrasound imaging with speed of sound-based phase correction: a numerical study," Phys. Med. Biol. 58(19), 6663-6681 (2013).

21. V. Periyasamy and M. Pramanik, "Monte Carlo simulation of light transport in tissue for optimizing light delivery in photoacoustic imaging of the sentinel lymph node," J. Biomed. Opt. 18(10), 106008 (2013).

22. S. L. Jacques, "Optical properties of biological tissues: a review," Phys. Med. Biol. 58(11), R37-R61 (2013).

23. Laser Institute of America, American National Standard for Safe Use of Lasers, ANSI Z136.1-2000, American National Standards Institute, Inc., New York (2000).

Yuanyuan Bai received her master's degree in optics from Zhejiang University in 2015. Currently, she is a research assistant at Shenzhen Institutes of Advanced Technology, Chinese Academy of Sciences. Her research interests include the implementation of photoacoustic computed tomography imaging system for the detection of diseases and developing new surgical guidance methods for clinical applications.
Bing Cong received his $\mathrm{PhD}$ in medical imaging system sciences from Kyoto University, Japan, in 2015. Then he received his postdoctoral training at the Graduate School of Medicine, Kyoto University for visualization technique of structural and functional bioinformation, for example, ultrasonic elasticity imaging and photoacoustic imaging. He joined Shenzhen Institutes of Advanced Technology, Chinese Academy of Sciences in 2017. His current research interests include the development and translational research of full-view photoacoustic imaging system.

Xiaojing Gong is an associate professor at Shenzhen Institutes of Advanced Technology, Chinese Academy of Sciences. He received his $\mathrm{PhD}$ in fine instruments and mechanics from the University of Science and Technology of China in 2007. He is currently focusing on the research of biophotonics, including photoacoustic endoscopy, optical coherence tomography/microscopy, terahertz biomedical imaging, etc.

Liang Song is a professor at Shenzhen Institutes of Advanced Technology, Chinese Academy of Sciences. He received his PhD in biomedical engineering from Washington University in Saint Louis. $\mathrm{He}$ is the founding director of The Research Lab for Biomedical Optics, Shenzhen Institutes of Advanced Technology and the director of the Shenzhen Key Lab for Molecular Imaging. He has authored more than 50 peer-reviewed journal articles and has been granted more than 10 patents in the field of photoacoustic imaging.

Chengbo Liu is an associate professor at Shenzhen Institutes of Advanced Technology, Chinese Academy of Sciences. He received both his bachelor's and PhD degrees from Xi'an Jiaotong University, each in 2012 in biophysics and 2007 in biomedical engineering. During 2009 to 2011, he was a visiting scholar at Duke University, working on tissue spectroscopy for early cancer diagnosis. His current research interests include multiscale photoacoustic imaging technology development and photoacoustic translational research. 